Astronomy Day in 1993 within the context of the International Space Year. The data that were collected will supply an importance reference set for the observations to be made this July during the impacts.

The possibility exists that the phenomena will not live up to the public's expectations for we cannot expect astrophysical forecasting to be as accurate as weather forecasts, even in these times of advanced techniques and extensive whole-Earth observation from satellites. However, we can be sure that the observational effort will contribute enormously to our understanding of planetary phenomena, especially to atmospheric physics and the interactions between particles in planetary magnetospheres.
Fig. 2 - A drawing (not to scale) illustrating the geometry of the collision between the comet SL-9 and the planet Jupiter. A wide variety of plasma and atmospheric phenomena will take place in the very dynamic environment of the Jovian system. (Courtesy of $R$. Prange, IAS, Orsay)

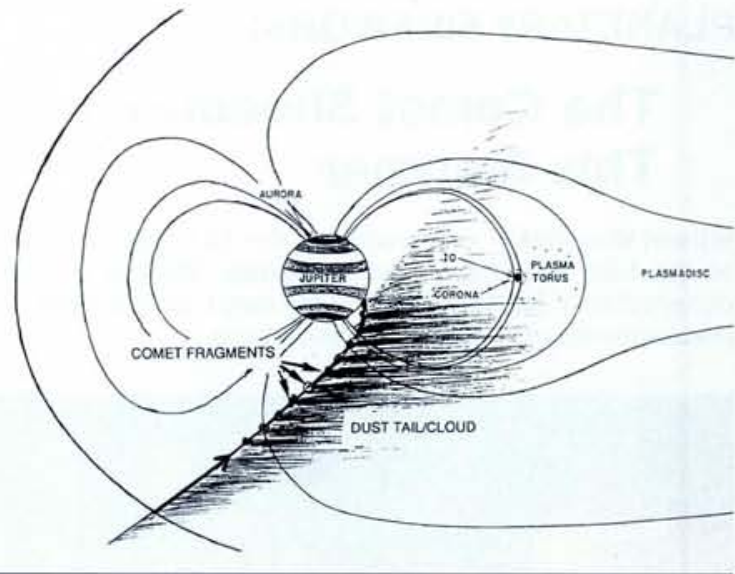

UNK, Protvino

\section{A Milestone in Spite of Hardships}

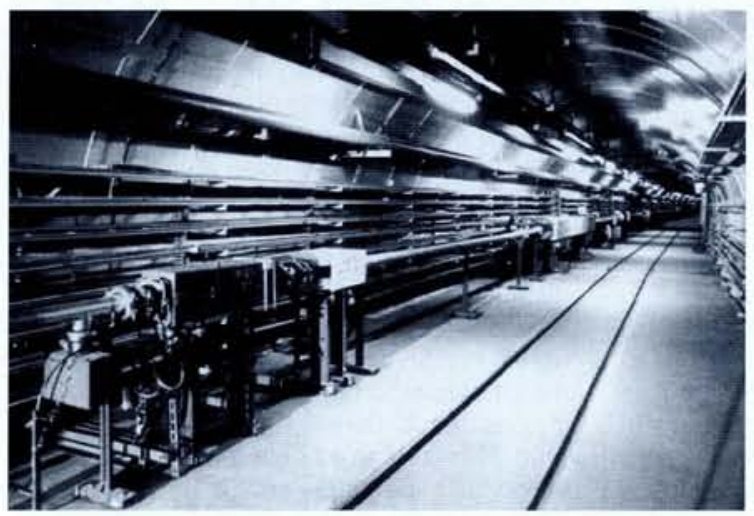

View of the UNK's transfer-line tunnel showing the beam-line magnets.

A typical control screen for the transfer line. At the top, a synoptic view of equipment in the beam line; at the centre, so-called "sliders" allowing adjustment of magnet currents; at the bottom, horizontal and vertical beam profiles as measured at points along the line. The control software was developed using Vsystem, a commercial controls package from Vista Control Systems, Inc., Los Alamos, USA.

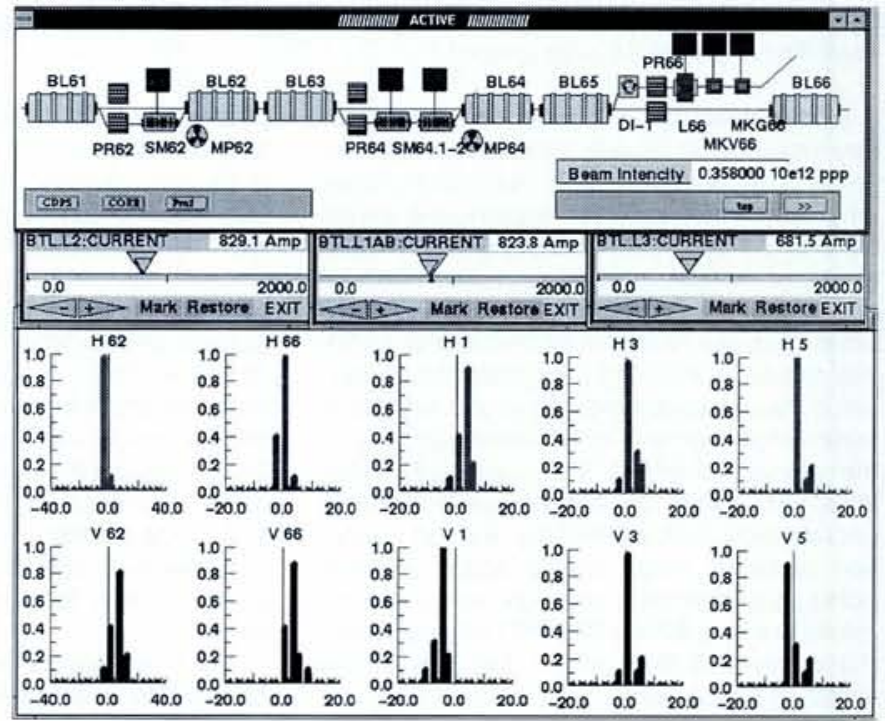

design value. The combination of beam diagnostics and control systems, which were in part developed in collaboration with CERN, allowed operation of the whole beam-transfer line from one central point in a user-friendly way.

Accelerator staff and particle physicists everywhere rejoice with their Russian colleagues in Protvino and hope that the milestone signifies a lasting positive turn in the UNK endeavour.

B. Kuiper, CERN that this transfer line be tried out with an actual proton beam well in advance of the accelerator itself, as was the case for CERN's LEP collider.

The exercise aimed to test two main aspects: first, the functioning of the design and the implementation of the beam ejection and transfer line and, second, the integration of the equipment into a coherent unit using the beam diagnostics and control systems. The $2.7 \mathrm{~km}$-long transfer line houses 52 dipole magnets of $5.8 \mathrm{~m}$ in length, 88 quadrupole lenses and 56 correction magnets. The beam diagnostics comprise 3 current monitors, 46 position monitors, 26 profile monitors as well as beam loss and halo monitors. The control system uses microprocessor-based equipment controllers, personal computers at an intermediate level and, as the operator interface, workstations with modern graphics software packages.

The injector synchrotron U70 accelerated 5 out of the possible 30 proton bunches at $6 \mathrm{MHz}$ to $65 \mathrm{GeV}$, at which energy the beam was recaptured at $200 \mathrm{MHz}$, as is required for UNK. A beam of about $3.5 \mathrm{x}$ $10^{11}$ protons per pulse was thus ejected into the transfer line and successfully steered up to a beam stopper placed at $2 \mathrm{~km}$ from the start. The emitance measured at that point was in good agreement with the

\section{Conference on EMERGENCE OF MODERN PHYSICS} Berlin, 20-24 March 1994

Organized by:

- History of Physics Division of the German Physical Society

- EPS Interdivisional Group on the History of Physics

- Commission on the History of Modern Physics of the IUHPS-

DHS

In conjunction with the celebration of the 150th Anniversary of the German Physical Society

To commemorate the centenary of the discovery of X-rays, the Zeeman effect, radioactivity, and the electron and to analyze the accompanying debates that shaped modern physics.

\section{Abstracts by 1 September 1994}

Contact: Fabio Bevilacqua, Dip. di Fisica, Univ. di Pavia,

Via Bassi, 6, I-27100 Pavia; tel./fax: +39-382-50 74 95 / 507563 